\title{
sciendo
}

Current Issues in Pharmacy and Medical Sciences

Formerly ANNALES UNIVERSITATIS MARIAE CURIE-SKLODOWSKA, SECTIO DDD, PHARMACIA

\section{Structural changes in liver under conditions of experimental hemorrhagic stroke}

\author{
Natalia Motorna*ic \\ O.O. Bogomolets National Medical University, Department of Histology and Embryology, T. Shevchenko 13, Kyiv, 01601, Ukraine
}

\begin{tabular}{l}
\hline ARTICLE INFO \\
\hline Received 31 May 2018 \\
Accepted 04 October 2018
\end{tabular}

Keywords:

liver,

stroke,

capillary sinusoids,

hepatocytes injury.

\begin{abstract}
The article describes the results of histological and morphometric studies of liver in Balb/c mice with hemorrhagic stroke. The hemorrhagic stroke was modeled in the animals by administering autoblood in volume of $0.1 \mathrm{ml}$ in the right hemisphere, and within 5,10 and 30 days an analysis of structural changes in the liver was performed. Progressive changes were established in terms of 5-10 days of the experiment. This consisted of changes in the sinusoidal capillaries and notable changes in the central veins of the liver lobuli. Herein, acute dilatation and erythrocytal stasis were most pronounced around the lumen of the central veins, while hepatocytes with signs of necrosis (severe cytoplasmic swelling, vacuolar dystrophic changes) were detected in the sinusoid capillaries. The results of the morphometry indicated an increase in the area of the nucleus and the cells caused by intracellular swelling, domination of euchromatin and decrease of total density of chromatin in nuclei. Partial regression of the diameter of sinusoidal capillaries and the area of hepatocytes were detected on the $30^{\text {th }}$ day of the experiment. The changes in the sinusoidal capillaries of the liver lobules are assessed as secondary to stroke, as well as to changes in organ microcirculation, and are associated with dystrophic changes in the hepatocytes.
\end{abstract}

\section{INTRODUCTION}

A stroke is not just a neurological disorder. It affects the functioning of other organs. Zhang et al. (2018) [1] report that liver disease and stroke have a negative correlation. In experimental studies, after 10 minutes of the simulation of stroke and hyperthermia, decrease in activity of superoxide dismutase and increase of free radicals in the liver were detected [2]. Moreover, two hours after brain ischemia/ reperfusion in rats with a fatty diet, a sharp decline in the activity of antioxidant enzymes (superoxide dismutase, glutathione peroxidase) and increased levels of malondialdehyde and free calcium in the liver were observed. Ischemia also increased the level of caspase-3, whose rate in stroke was lower compared with diet [3]. The authors note that simulation of ischemia of the brain in rats dramatically exacerbates metabolic parameters and liver damage. This is explained by the production of free radicals and the reduction of enzymatic antioxidant defense mechanisms [2]. In analytical publications, this was considered to have come about by the activation of the synthesis of liver enzymes

\footnotetext{
* Corresponding author

e-mail: natalivfrcbv@gmail.com
}

as a response to the excessive release of cytotoxic neurotransmitters by ischemic brain tissue, in particular, glutamate [4]. Most scientific publications are, however, devoted to the study of molecular markers of liver function against ischemia, and the structural foundations of these changes remain poorly researched. Hence, further research is needed to clarify the mechanisms underlying the link between the stroke and changes in the structure of the liver. In this study, we put forward the hypothesis that on the background of a hemorrhagic stroke, morphological changes in the liver can occur as a manifestation of structural and functional changes in the body.

The aim - to study the histological changes of the liver against a background of hemorrhagic stroke.

\section{MATERIALS AND METHODS}

The study was conducted on laboratory mice of the Balb/c lineage with an average weight of 18-20 g. Animals were divided into 4 groups of 10 animals: group 1 - control group (intact mice), group 2 - animals on the $5^{\text {th }}$ day of stroke; group 3 - animals on the $10^{\text {th }}$ day of stroke; group 4 - animals on the $30^{\text {th }}$ day of stroke. The model of intracerebral 
hematoma was used to simulate a stroke. Reproduction of limited hemorrhage of the brain of animals was achieved by administration of $0.15-0.2 \mathrm{ml}$ of autoblood in the right hemisphere $(\mathrm{L}=1.5 ; \mathrm{H}=3.0 ; \mathrm{AP}=1.0)[5,6]$. In all animals, the hematoma was localized in the right inner capsule (capsula interna dextra) of the brain.

For the histological examination, the liver was fixed in $10 \%$ neutral formalin (0.1 M cold PBS, pH 7.4). Dehydration was carried out according to the standard method of ascending concentrations of ethanol, clarified in dioxane and xylene. As an intermediate between xylene and paraffin, a mixture of xylene paraffin was used 1: 1. Impregnation in paraffin was carried out in a thermostat ST-50C at temperatures of $37^{\circ} \mathrm{C}$ and $56^{\circ} \mathrm{C}$ (UOSlab, Ukraine). The liver samples were contained in paraplast (Leica Surgipath Paraplast Regular). Histologic sections were obtained on a microtome Thermo Microm HM 360 Rotary Microtome. Sections with a thickness of 5 microns were then stained with hematoxylin and eosin. General morphological organization of the organ was examined on histological preparations. In addition, we assessed the state of the blood vessels of the liver lobes (central vein, sinusoidal capillaries, interstitial arteries and veins), changes in hepatocytes (hypertrophy of cells, nuclei hypertrophy, the appearance of atypical nuclei, karyopicnosis and lithic changes), as well as the presence and density of nidi of leukocyte infiltrates. The morphometric analysis was performed using an Olympus BX 51 microscope and CarlZeiss software (AxioVision SE64 Rel.4.9.1).
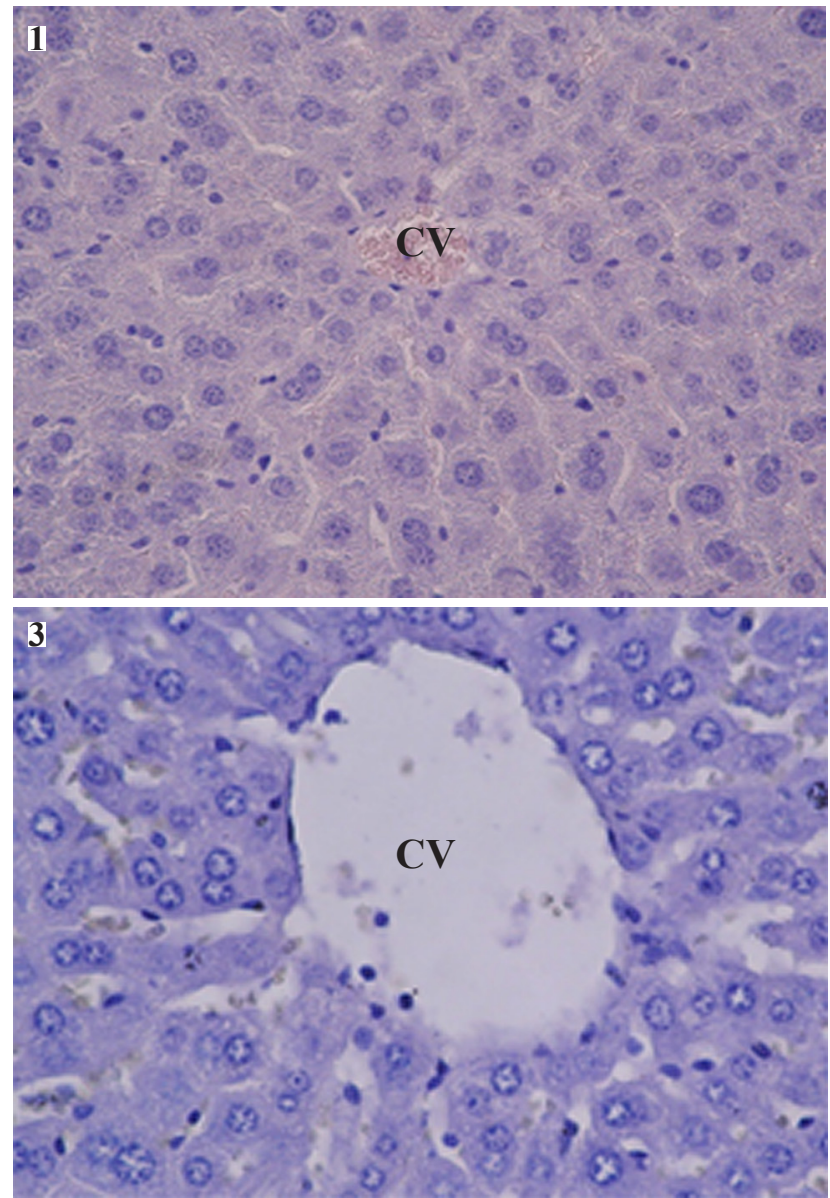

The statistical estimation was carried out according to the nonparametric criterion of Kruskal-Wallis. Data samples were analyzed using Origin Lab software version 8.0. The difference was considered statistically significant at $\mathrm{p}<0.05$.

All laboratory (experimental into groups, animal fixation, anesthesia, blood from caudal artery) procedures were carried out in the Bogomolets National Medical University in accordance with the U.K. Animals (Scientific Procedures) Act, 1986 and related guidelines, EU Directive 2010/63/EU for animal experiments, and the National Institutes of Health Guide for the care and use of laboratory animals (NIH Publications No. 8023, revised 1978). The research was approved by the Bioethical Committee for Human Subjects or Animal Research at Bogomolets National Medical University, Minutes No. 100, 21 February, 2017.

\section{RESULTS}

In the experimental study, no fatal cases occurred. However, changes were observed on histological preparations of liver. These indicated a violation of regional microcirculation. Sinusoid capillaries and especially the central vein of the classical lobuli were sharply dilated (Fig. 1). Diameter of the capillaries was statistically significantly increased $(\mathrm{p}<0.05)$. Erythrocyte stasis was also noted. In some capillaries, isolated leukocytes and macrophages were detected. The most pronounced changes were established centrolobularly around the central veins.
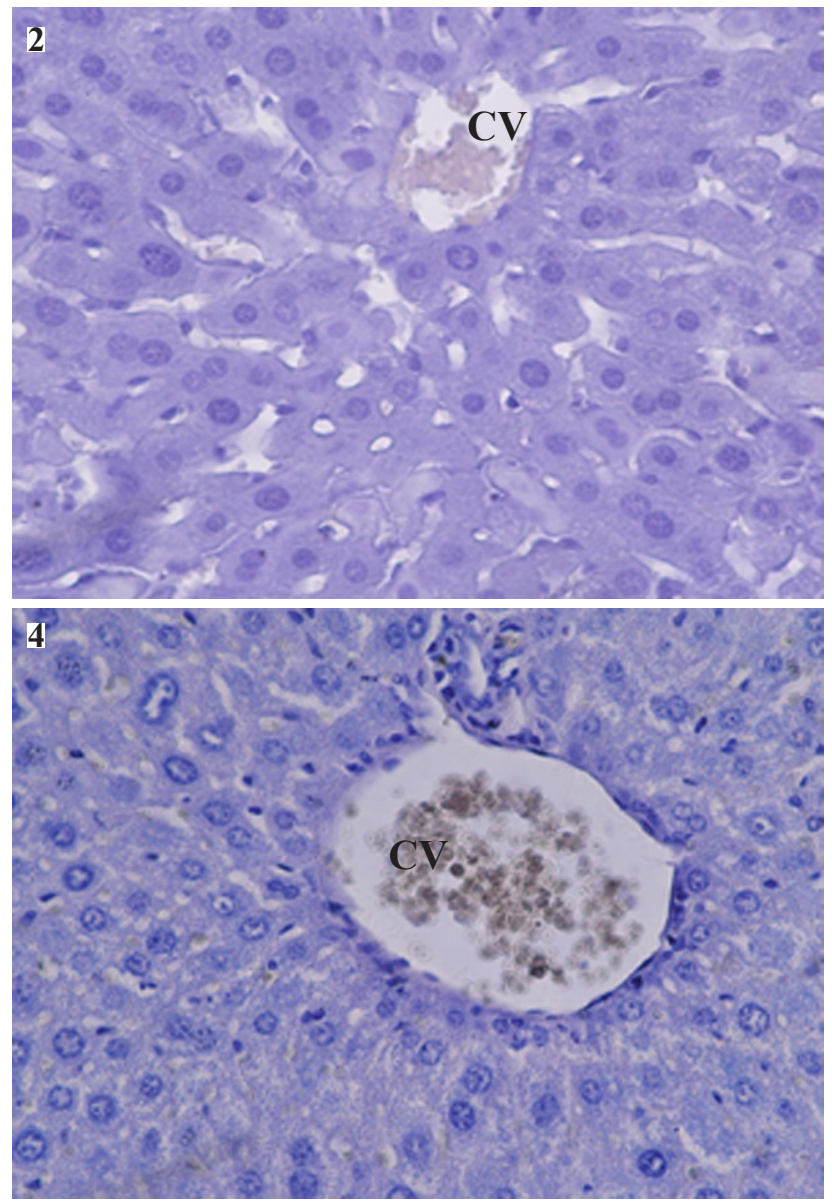

Note: 1 - control group (intact mice); 2 - animals on the $5^{\text {th }}$ day of stroke; 3 - animals on the $10^{\text {th }}$ day of stroke; 4 - animals on the $30^{\text {th }}$ day of stroke, $\mathrm{CV}-$ central vein. Hematoxylin-eosin, $\times 400$

Figure 1. Histological structure of the liver of the control and experimental groups 
Structural changes differed depending on the terms of experiment. Thus, on the $5^{\text {th }}$ day, dilatation of sinusoidal capillaries was detected in $90 \%$ of cases, and dilatation of central veins in $100 \%$ of investigated animals. Erythrocyte stasis was noted in the majority of hepatic lobules. The stasis of the formed elements was not detected in the central veins. Signs of hepatocyte necrosis were not detected, but cell morphology had a significant variability, and hypertrophy of the nuclei of individual hepatocytes was marked. This should be evaluated as a manifestation of functional activation against the background of the pathological process. In $30 \%$ of all the animals, an increase in the number of monocyte cells (monocytes, macrophages) was observed in the sinusoidal capillaries.

On the $10^{\text {th }}$ day of experiment, progressive dilatation of sinusoidal capillaries was established, a sharp increase in diffusion of the lumen of the capillaries was noted in all the lobes of the samples under study. Hepatocytes with signs of necrosis (severe cytoplasmic edema, vacuolar dystrophic changes) were also observed around the central vein and in the pericentral parts of the lobules. Cellular derivates (detritus of dead cells) were recorded in the lumen of the microvessels.

On the $30^{\text {th }}$ day of observation, signs of regression of structural changes in the liver were observed. These consisted of lumen reduction in the hemocapillaries, as well as a partial reduction of the lumen of the central veins of the lobules. Signs of dilatation of capillaries were found in 50\% of all the animals, and dilatation of central veins of liver lobules in $100 \%$. Hepatocytes with hypertrophied nuclei were also registered, however, most cells approached the control group with cytological signs of the nucleus. Signs of inflammatory reaction were not detected during 10 days of observation.

The dynamics of structural changes in the liver are clearly illustrated by the results of the morphometric study. Herein, morphometric indices (area of the nucleus and soma of hepatocytes, diameter of hemocapillaries) were significantly increased in groups 2 and 3, that is, in terms of observation of the $5^{\text {th }}$ and $10^{\text {th }}$ days after stroke modeling $(\mathrm{p}<0.05)$ (Table 1$)$. The data in-group 4 indicate a partial regression of structural changes in the terms of 5 and 10 days. Moreover, the difference between groups 1 and 4 was also found $(p<0.05)$, indicating the continuation of reactive organ changes in an experimental hemorrhagic stroke. Structural rearrangements of stromal elements of the liver lobules were not detected in any of the studied samples.

Table 1. Morphometric parameters of the liver of the control and experimental groups

\begin{tabular}{|c|c|c|c|c|}
\hline № & Group & $\begin{array}{c}\text { Area } \\
\text { of hepatocyte, } \\
\mu \mathrm{m}^{2}\end{array}$ & $\begin{array}{c}\text { Area } \\
\text { of hepatocyte } \\
\text { nucleus, } \mu \mathrm{m}^{2}\end{array}$ & $\begin{array}{c}\text { Diameter } \\
\text { of sinusoidal } \\
\text { capillaries, } \mu \mathrm{m}\end{array}$ \\
\hline 1 & $\begin{array}{l}\text { Control group } \\
\text { (intact mice) }\end{array}$ & $360.40 \pm 0.94$ & $68.06 \pm 0.05$ & $9.08 \pm 0.17$ \\
\hline 2 & Stroke, $5^{\text {th }}$ day & $521.33 \pm 1.35^{*}$ & $104.06 \pm 0.34 *$ & $12.85 \pm 0.26 *$ \\
\hline 3 & Stroke, $10^{\text {th }}$ day & $599.48 \pm 1.47^{* \wedge}$ & $114.92 \pm 0.25^{* \wedge}$ & $14.75 \pm 0.26 * \wedge$ \\
\hline 4 & Stroke, $30^{\text {th }}$ day & $456.78 \pm 1.30 * \#$ & $80.73 \pm 0.24 * \#$ & $11.00 \pm 0.22^{* \wedge \#}$ \\
\hline
\end{tabular}

\section{DISCUSSION}

The analytical studies revealed an association of liver diseases with risk of stroke [7]. Liver disruption appeared more strongly with worse prognosis after intracerebral hemorrhage and ischemic stroke. The authors did not detected difference in the association between liver disease and stroke subtype.

From literary sources, in patients with traumatic and ischemic brain damage, changes of biochemical parameters of liver function are seen [8-10]. However, although dyslipidemia increases the risk of ischemic and hemorrhagic stroke [11], studies have generated controversial data on the relationship between lipid levels and severity of stroke [12]. In women, for example, it was found that lipid parameters were not associated with the severity of stroke, while in men, the reduction of triglycerides and LDL was associated with a worse prognosis of the disease [11]. In other studies, the changes in some of the biochemical parameters that may be mediated indicated a disorder of the body. Here, an increase in the level of bilirubin and the association with the severity of a stroke was revealed [13]. Furthermore, changes in liver enzymes (superoxide dismutase), cytotoxic products of lipid peroxidation and bilirubin in the acute phase of a stroke were directly or indirectly related to the size of the site of the brain infarction. Thus, in the acute phase of stroke, statistically, the level of liver-associated enzymes (glutamate oxaloacetate transaminase, glutamate piruvate transaminase, gamma glutamyl transferase) were enhanced [4]. But liver enzymes are non-specific markers of cytotoxic lesions, including brain damage, hemolysis, and muscle atrophy. Therefore, indicators of enzymes alone cannot reliably testify to dysfunction of the liver.

The results of recent histological studies and experiments (in particular) make it possible to understand the structural changes in the liver and its secondary injuries that come about through stroke. A stroke causes activation of inflammatory cascades, resulting in a systemic inflammatory reaction and the consequence of secondary liver dysfunction. In our work, the initiation of inflammatory response was detected only on the $5^{\text {th }}$ day of the experiment. This can be considered an acute period of the pathological process. But subsequently, such changes were not registered, that is, there was a regression or completion of the immune response. The inflammatory response and even liver cell apoptosis can, hence, be an independent source of oxidative stress and can be considered a key mechanism of liver damage. These changes are secondary to the source of ischemia and are likely to be a systemic, albeit nonspecific response of the liver to damage.

Histological studies may explain the cause of such a reaction, but the results of such work are only described in the literature in a fragmentary way. In particular, in some articles, an enlargement of the biliary ducts of the liver was reported in patients with stroke and traumatic brain damage $[14,15]$. The publication [16] describes necrosis and apoptosis of hepatocytes in the periarterial zone, damage to the endothelial cells of the capillaries, focal thrombosis of the lumbar vessels and infiltration of the macrophages after traumatic brain damage. Other authors, however, report no changes 
in biliary tract and bilirubin levels during the first 7 days of the disease [4].

Thus, the local response of immunocompetent cells must develop much faster. In the liver, we found that the level of MPC-1, TNF- $\alpha$ and CD68-positive macrophages was already increased already as of the $1^{\text {th }}$ day after stroke [17]. Thus, we propose that infiltrated leukocytes and activated resident macrophages are a major proinflammation factor of secondary injury of liver. Still, morphological data on this subject are very limited.

Our results also showed centrolobular dystrophic changes in hepatocytes and sharp dilation of sinusoidal capillaries. Certain authors hold the opinion that violation of the autonomic nervous system influences the regulation of microcirculation and bile duct and, as a result, structural and functional changes in the organ [4]. Other publications explain the association of changes in liver microcirculation with HSV-I infection [18,19], stroke [20] and stroke-induced immune response [21]. On the basis of our research, it was concluded that the stroke is not limited to cerebral circulation, brain injury or immune system disruption, it affects changes in the blood flow of the liver and is a factor in secondary post-stroke liver damage.

We agree that our study is limited and focused only on morphological data, but own results sufficiently confirm that changes in the liver occur and can explain the functional changes of organ. Further research about the association of stroke and liver injury may lead to improve understanding of the development of liver diseases and their treatment.

\section{CONCLUSION}

The studies showed that stroke is not limited to brain disorders, it affects the morphological parameters of the liver. The main manifestation of structural changes in the liver was the dynamic changes in the microcirculation of the liver lobules. This consisted in increases in the lumen of the sinusoidal capillaries on the $5^{\text {th }}$ and $10^{\text {th }}$ days and a partial regression on the $30^{\text {th }}$ day. Herein, the erythrocytic stasis of the capillaries and the initiator signs of the inflammatory reaction that occurred resulted in dystrophic changes in the hepatocytes in the period of 30 days. Thus, the damage to the hepatocytes was assessed as secondary to stroke changes associated with changes in organ microcirculation.

\section{ORCID iDs}

Natalia Motorna (Dhttps://orcid.org/0000-0002-7038-755X

\section{REFERENCES}

1. Zhang X, Qi X, Yoshida EM, Méndez-Sánchez N, Hou F, Deng H, Wang $\mathrm{X}$, et al. Ischemic stroke in liver cirrhosis: epidemiology, risk factors, and in-hospital outcomes. Eur J Gastroenterol Hepatol. 2018; 30(2):233-40.

2. Yang CY, Lin MT. Oxidative stress in rats with heatstroke-induced cerebral ischemia. Stroke. 2002;33(3):790-4.

3. Gong W-H, Zheng W-X, Wang J, Chen S-H, Pang B, Hu X-M, Cao $\mathrm{X}-\mathrm{L}$. Coexistence of hyperlipidemia and acute cerebral ischemia/ reperfusion induces severe liver damage in a rat model. World Journal of Gastroenterology. WJG. 2012;18(35):4934-43.
4. Muscari A, Collini A, Fabbri E, Giovagnoli M, Napoli C, Rossi V, et al. Changes of liver enzymes and bilirubin during ischemic stroke: mechanisms and possible significance. BMC Neurology. 2014;14:122.

5. Savosko SI, Chaikovsky JuB, Pogorela NKh, Makarenko AN. Features of histostructural changes in rat cerebral cortex in hemorrhagic stroke modeling. International Journal of Physiology and Pathophysiology. 2013;4(2):113-21.

6. Gumenyuk AV, Motorna NV, Rybalko SL, Savosko SI, Sokurenko LM, Chaikovsky YuB. Mutual influence of herpes virus infection activation and cerebral circulation impairment on the state of brain cells. Biopolym Cell. 2016;32(2):126-30.

7. Parikh NS, Merkler AE, Schneider Y, Navi BB, Mamel H. Dischange disposition after stroke in patients with liver disease. Stroke. 2017;48(2):476-8

8. Meythaler JM, Hazlewood J, DeVivo JM, Rosner M. Elevated liver enzymes after nontraumatic intracranial hemorrhages. Arch Phys Med Rehabil. 1998;79:766-71.

9. Abdeldyem SM, Goda T, Khodeir SA, Abou Saif S, Abd-Elsalam $S$. Nonalcoholic fatty liver disease in patients with acute ischemic stroke is associated with more severe stroke and worse outcome. J Clin Lipidol. 2017;11(4):915-9.

10. Tziomalos K, Giampatzis V, Bouziana SD, Spanou M, Papadopoulou M, Pavlidis A, et al. Association between nonalcoholic fatty liver disease and acute ischemic stroke severity and outcome. World Journal of Hepatology. 2013;5(11):621-6.

11. Cuadrado-Godia E, Jiménez-Conde J, Ois A, Rodríguez-Campello A, García-Ramallo E, Roquer J. Sex differences in the prognostic value of the lipid profile after the first ischemic stroke. J Neurol. 2009;256(6):989-95.

12. Tziomalos K, Giampatzis V, Bouziana SD, Spanou M, Kostaki S, Papadopoulou M, et al. Prognostic significance of major lipids in patients with acute ischemic stroke. Metab Brain Dis. 2017; 32(2):395-400.

13. Pineda S, Bang OY, Saver JL, Starkman S, Yun SW, Liebeskind DS, et al. Association of serum bilirubin with ischemic stroke outcomes. J Stroke Cerebrovasc Dis. 2008;17:147-52.

14. Sevastos N, Savvas SP, Rafailidis PI, Manesis EK. Cholestasis in acute stroke: an investigation on its prevalence and etiology. Scand J Gastroenterol. 2005;40:862-6.

15. Sanfilippo F, Veenith T, Santonocito C, Vrettou CS, Matta BF. Liver function test abnormalities after traumatic brain injury: is hepatobiliary ultrasound a sensitive diagnostic tool? British Journal of Anaesthesia. 2014;112(2):298-303.

16. Zhu R, Guo W, Fang H, Cao S, Yan B, Chen S, et al. Kupffer cell depletion by gadolinium chloride aggravates liver injury after brain death in rats. Molecular medicine reports. 2018;17:6357-62.

17. Wang YY, Lin SY, Chuang YH, Sheu W HH, Tung KC, Chen CJ. Activation of hepatic inflammatory pathways by catecholamines is associated with hepatic insulin resistance in male ischemic stroke rats. Endocrinology. 2014;155(4):1235-46.

18. Motorna N, Rybalko S, Kvitnitskaya-Ryzhova T, Starosyla D, Strokina I, Kaminsky R, et al. Ultrastructural changes in murine liver following HSV infection and stroke. Ultrastructural changes in murine liver following HSV infection and stroke. Curr Issues Pharm Med Sci. 2018;31(1):10-12.

19. Motorna NV, Rybalko SL, Sokurenko LM, Starosyla DB, Savosko SI, Chaikovsky YuB. Patterns of herpetic infection reactivation in the liver. Microbiology Journal. 2017;79(5):70-9.

20. Gumenyuk A, Motorna N, Rybalko S, Savosko S, Sokurenko L, Starosyla D, et al. Development of herpetic infection associated with stroke and its correction with acyclovir. Curr Issues Pharm Med Sci. 2017;30(1):20-3.

21. Motorna NV, Rybalko SL, Starosyla DB, Guzyk MM, Strokina IG, Kaminsky RF, et al. The study of leukocyte phagocytic activity in the presence of herpetic infection and stroke. Wiad Lek. 2018;71 (1 pt 2):155-9. 